\title{
EELS Studies on Nanodiamonds and Amorphous Diamond-like Carbon Materials
}

\author{
R. Arenal ${ }^{1,2}$ \\ ${ }^{1}$ Laboratorio de Microscopias Avanzadas (LMA), Instituto de Nanociencia de Aragon (INA), \\ Universidad de Zaragoza, Calle Mariano Esquillor, 50018 Zaragoza, Spain. \\ ${ }^{2}$ ARAID Fundation, Calle Mariano de Luna, 50018 Zaragoza, Spain.
}

Chemical analyses at the local (sub-nanometer/atomic) scale are crucial for improving the understanding of materials. For instance, for doped carbon nanostructures (as those of diamond family), these analyses are critical because their properties strongly depend on the chemical environment/configuration of the dopants [1-3]. Electron energy-loss spectroscopy (EELS) is an essential and powerful technique to perform such local chemical analyses [1-4].

In this contribution, we will present a detailed study of the structure and local composition of nitrogen-doped ultrananocrystalline diamond (UNCD) films [5-8], hydrogenated amorphous diamond-like carbons [9] and nano-/micro-crystalline diamond films [10]. These studies have been developed combining HR(S)TEM, EELS and micro-Raman multi-wavelength spectroscopy. Regarding the EELS analyses, we will revisit the procedures to extract proper and reliably quantitative chemical information from these spectra.

Fig. 1 (a) corresponds to a HRTEM image of an UNCD film sample. In this micrograph, an elongated diamond nanocrystal (called nanowires (NWs) [5-8]) is displayed. Under normal process conditions, these UNCD films are highly electrically insulating, but they can become highly conducting when Ar is substituted in the synthesis gas with some of $\mathrm{N}_{2}[1,5]$. The formation of these NWs starts to appear when the $\mathrm{N}_{2}$ content in the gas phase reaches about $10 \%$ in volume. From these studies, we concluded that the insulator-metal transition of these films is strongly correlated with the formation of these diamond NWs. Indeed, these NWs are enveloped by a $\mathrm{sp}^{2}$-based carbon layer (Fig. 1 (a)) that seems to provide the conductive path for electrons [5-8]. Thus, here we will present the local EELS analyses on these samples (Fig. 1 (b)-(c)), focusing on the analyses of the $\mathrm{N}$ atomic configurations [8].

For studying complex materials composed by a mixture of $\mathrm{sp}^{3} / \mathrm{sp}^{2}$ configurations, we have developed a new method to determine the fraction of these configurations from the EELS spectra, see Fig. 2 (a). This method does not require specific experimental setup and can include contributions from heterospecies $[9,10]$. Thus, our results provide a complete combination of Chybridization and spatial elemental analyses for shedding light on hydrogenated amorphous diamond-like carbon films. In particular, we will show how the deposition process induces a gradient of $\mathrm{sp}^{2}$ ratio in the thin films and how this gradient is modified as a function of the annealing time [9].

Finally, we will present our recent results on nano- and micro-crystalline diamond films [10]. We have shown strong in-depth inhomogeneities of the local chemistry in these films and we have investigated the effects of methane's excess on the evolution of the morphology, nanostructure and composition of the films [10].

In summary, these studies elucidate crucial questions concerning the local composition (atomic configuration) of these materials. This detailed knowledge is essential for better understanding the outstanding properties of such materials. In addition, these works clearly demonstrate the pertinence 
of combining several local and macroscopic techniques to extract properly and reliably this kind of information.

\section{References}

[1] O.A. Shenderova \& D.M. Gruen, UNCD: synthesis, properties and applications. William Andrew. (2012).

[2] R. Arenal, X. Blase, A. Loiseau, Adv. Phys. 59, 101 (2010).

[3] R. Arenal, Review paper, submitted.

[4] R.F. Egerton, EELS in the Electron Microscope. Springer Science \& Business Media (2011).

[5] R. Arenal, P. Bruno, D.J. Miller, M. Bleuel, J. Lal, D.M. Gruen, Phys. Rev. B 75, 195431 (2007).

[6] R. Arenal, O. Stephan, P. Bruno, D.M. Gruen, Appl. Phys. Lett. 94, 111905 (2009).

[7] R. Arenal, G. Montagnac, P. Bruno, D.M. Gruen, Phys. Rev. B 76, 245316 (2007).

[8] R. Arenal and D.M. Gruen, to be submitted.

[9] L. Lajaunie, C. Pardanaud, C. Martin, P. Puech, C. Hu, M.J. Biggs, R. Arenal, Carbon 112, (2017).

[10] J.G. Buijnsters, C. Pardanaud, L. Vázquez, R. Gago, J.L. Endrino, L. Lajaunie, R. Arenal, in preparation. [11] Many thanks to L. Lajaunie, O. Stephan, D.M. Gruen, P. Bruno, C. Parpanaud and J.G. Buijnsters who contributed to different parts of these works. The research leading to these results has received funding from the Spanish MINECO (MAT2016-79776-P) and from the EU H2020 programs ETN Grant Agreement 642742 and "Graphene Flagship" Grant Agreement 696656.
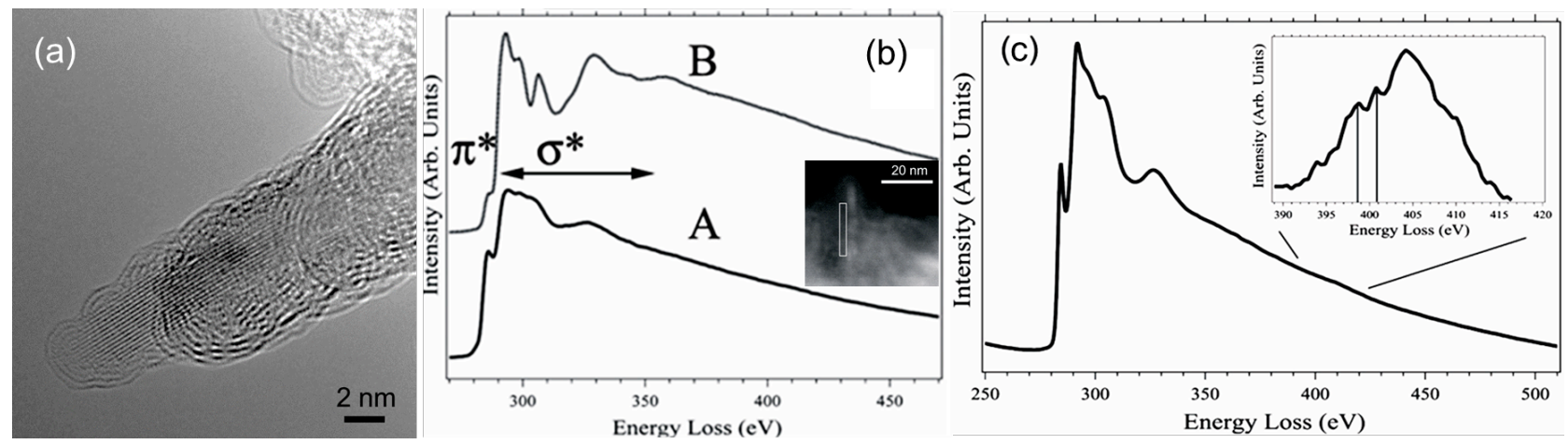

Figure 1. N-doped UNCD materials: (a) HRTEM image showing a diamond nanocrystal covered by a $\mathrm{sp}^{2}$ C sheath. (b) STEM-EELS spectra recorded on two different positions of an UNCD film: "A" corresponds to $\mathrm{sp}^{2}$ bounded $\mathrm{Cs}$; " $\mathrm{B}$ " (middle of a NW) is assigned to $\mathrm{sp}^{3} \mathrm{C}$ with a small contribution of $\mathrm{sp}^{2}$ bounded carbon from the surface of the NW. (c) STEM-EELS spectra acquired at the surface of another NW. The $\mathrm{N}-\mathrm{K}$ edge is observed in this spectrum and it has been analysed (insert). (b) \& (c) are adapted from [5, 6].
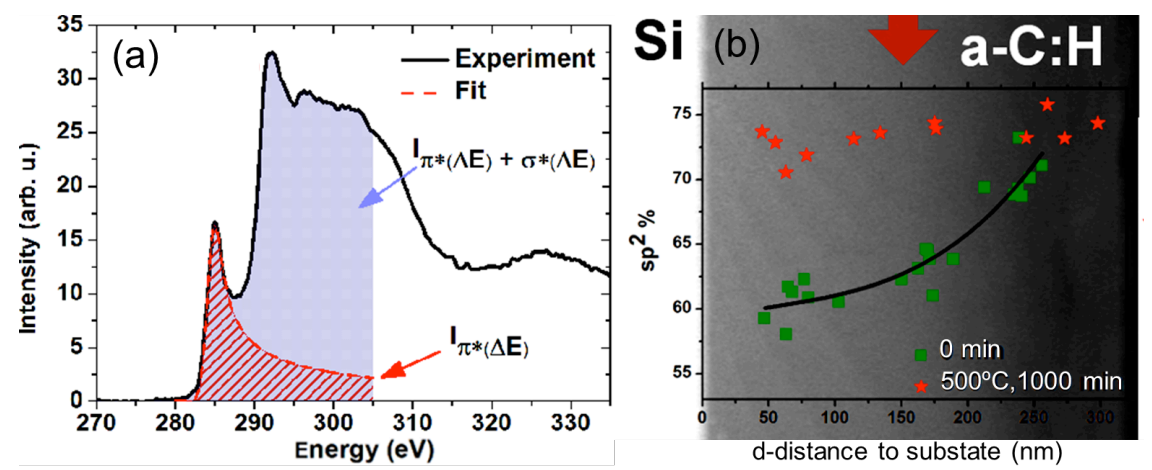

Figure 2. - Revisiting EELS studies on hydrogenated amorphous carbon materials: (a) EELS spectra of a graphite sample illustrating the method used to determine the $\mathrm{R}$ ratio (parameter related to $\mathrm{sp}^{2} / \mathrm{sp}^{3}$ content). (b) Variation of the $\mathrm{sp}^{2}$ fraction, determined by EELS, as a function of the distance between the electron probe and the substrate. The black line is a guide for the eyes. Adapted from [9]. 